\title{
Changes of the eSheath Outer Dimensions Used for Transfemoral Transcatheter Aortic Valve Replacement
}

\author{
Till Koehler, Michael Buege, Heinrich Schleiting, Melchior Seyfarth, \\ Klaus Tiroch, and Marc Vorpahl \\ Department of Cardiology, Zentrum für Forschung in der Klinischen Medizin (ZFKM), HELIOS Klinikum Wuppertal, \\ Herzzentrum, University Witten/Herdecke, Arrenberger Straße 20, 42117 Wuppertal, Germany
}

Correspondence should be addressed to Marc Vorpahl; marc.vorpahl@helios-kliniken.de

Received 31 October 2014; Accepted 25 March 2015

Academic Editor: Namal Wijesinghe

Copyright (C) 2015 Till Koehler et al. This is an open access article distributed under the Creative Commons Attribution License, which permits unrestricted use, distribution, and reproduction in any medium, provided the original work is properly cited.

\begin{abstract}
Innovative catheter systems with lower-profile sheaths and a dynamic expansion mechanism (DEM) were recently introduced for transcatheter aortic valve replacement (TAVR). However, the labeling of $14 \mathrm{~F}$ and $16 \mathrm{~F}$ eSheaths denote the inner nominal diameter. Exact changes of the clinically relevant outer diameters during usage are not available. eSheaths were measured every $30 \mathrm{~mm}$ using a digital caliper. Unused $14 \mathrm{~F}$ and $16 \mathrm{~F}$ eSheaths served as controls. Maximum eSheath diameters were measured after insertion of the Edwards Commander Delivery System (ECDS) into $14 \mathrm{~F}$ and $16 \mathrm{~F}$ eSheaths. Finally, eSheaths were retrieved and measured after TAVR. Outer diameters of control $14 \mathrm{~F}$ eSheaths were $5.8 \mathrm{~mm}$ and $6.50 \mathrm{~mm}$ for the $16 \mathrm{~F}$ eSheath. Introduction of the $23 \mathrm{~mm}$ and $26 \mathrm{~mm}$ ECDS into $14 \mathrm{~F}$ eSheaths showed a maximum diameter of $7.65 \mathrm{~mm}$ and $7.64 \mathrm{~mm}(P=\mathrm{NS})$. Introduction of the $29 \mathrm{~mm}$ ECDS into the $16 \mathrm{~F}$ eSheath showed the greatest diameter of $8.18 \mathrm{~mm}(P=0.03)$. After TAVR, diameters of the $14 \mathrm{~F}$ eSheaths were $7.14 \mathrm{~mm}$ (23 mm valve) and $7.26 \mathrm{~mm}$ (26 mm valve) $(P=\mathrm{NS})$, while $16 \mathrm{~F}$ eSheaths were $8.10 \mathrm{~mm}(29 \mathrm{~mm}$ valve $)(P \leq 0.03)$. Nominal $14 \mathrm{~F}$ and $16 \mathrm{~F}$ eSheaths showed a significant increase of the outer diameter during advancement of the ECDS and after TAVR implantation.
\end{abstract}

\section{Introduction}

Vascular complications contribute significantly to the morbidity and mortality during and after transcatheter aortic valve replacement (TAVR) via femoral arterial access in randomized trials and daily practice [1-7]. Catheter systems are continuously optimized including lower-profile sheath designs $[2,3]$. The current generation eSheath for the Edwards SAPIEN 3 TAVR is a nominal $14 \mathrm{~F}(\sim 4.7 \mathrm{~mm})$ or $16 \mathrm{~F}(\sim 5.3 \mathrm{~mm})$ femoral sheath with a dynamic expansion mechanism (DEM). This sheet system expands transiently during the passage of the SAPIEN 3 transcatheter heart valve and abates to a lower-profile diameter thereafter. The DEM facilitates the placement of the large femoral sheath in the frail TAVR population with atheroma-altered iliacofemoral vessels, vascular calcification burden, and vessel tortuosity and may therefore reduce adverse events at the vascular access site.

The manufacturer recommends a minimal vessel size of $5.5 \mathrm{~mm}$ for the $14 \mathrm{~F}$ eSheath $(23 \mathrm{~mm}$ and $26 \mathrm{~mm}$ Sapien 3 valve) and $6.0 \mathrm{~mm}$ for the $16 \mathrm{~F}$ eSheath $(29 \mathrm{~mm}$ Sapien 3 valve). However, the actual changes of the eSheath dimensions during the Sapien 3 transfemoral TAVR procedure are not published. Therefore, we performed an assessment of the outer diameter changes of the eSheath system before usage, during passage of the pusher through the ECDS system ex vivo and after the removal of the in vivo used eSheath. These measurements of the outer luminal diameters may help the TAVR operators to better understand the effective external dimensions of the current eSheath system during implantation.

\section{Methods}

2.1. eSheath Collection. First, $14 \mathrm{~F}$ eSheaths $(n=5)$ and $16 \mathrm{~F}$ eSheaths $(n=5)$ were analyzed before usage as controls; Edwards Lifescience Irvine CA, USA (Figure 1(a)).

Second, unexpanded control eSheaths were used for further ex vivo measurements including advancement of the ECDS. 


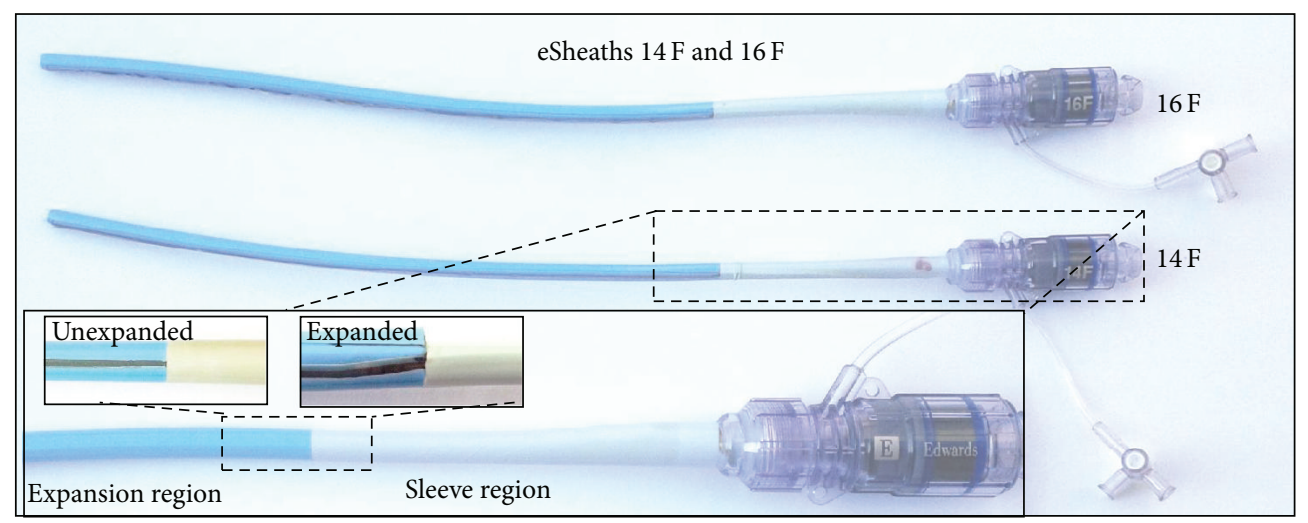

(a)

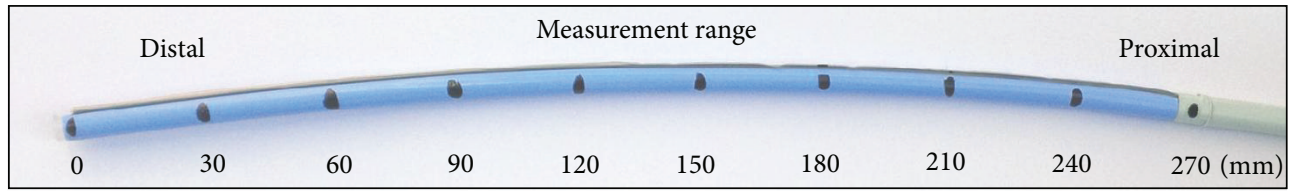

(b)

Figure 1: $14 \mathrm{~F}$ and $16 \mathrm{~F}$ femoral eSheath for Edwards Sapien 3 TAVR. (a) The eSheath system expands transiently during the passage of the Sapien 3 transcatheter heart valve and abates to a lower-profile diameter thereafter. A circumferential plastic sleeve stabilized the expandable part of the eSheath at the intersection between the expandable part and the stiff bleed back prevention valve. (b) The outer diameter of the expandable part of the eSheath was measured by a digital caliper from the most distal part $(0 \mathrm{~mm})$ in 30 mm increments until the sleeve region $(300 \mathrm{~mm})$ was reached.

Third, a total of 15 eSheaths were flushed with buffered saline and analyzed before disposal after femoral Sapien 3 transcatheter valve implantation. Five $14 \mathrm{~F}$ eSheaths were analyzed after implantation of $23 \mathrm{~mm}$ Sapien 3 valves, five $14 \mathrm{~F}$ eSheaths were analyzed after implantation of $26 \mathrm{~mm}$ Sapien 3 valves, and five $16 \mathrm{~F}$ eSheaths were analyzed after implantation of $29 \mathrm{~mm}$ Sapien 3 valves.

The study was approved by the local ethics committee and all patients provided written informed consent.

2.2. Digital Caliper. Measurements were performed using an ISO certified iGaging ABSOLUTE ORIGIN metric digital electronic caliper meeting DIN Standard 862 (resolution: $0.0005^{\prime \prime} / 0.01 \mathrm{~mm}$; accuracy: $0.001^{\prime \prime} / 0.02 \mathrm{~mm}$; range: $\left.6^{\prime \prime} / 150 \mathrm{~mm}\right)$, San Clemente, CA, USA. The caliper was calibrated to zero before each measurement.

2.3. eSheath Diameter Analyses. The first assessment focused on the unused $14 \mathrm{~F}$ and $16 \mathrm{~F}$ eSheaths, defined as controls ( $n=5$ for each group) (Figure 2(a)). We measured the outer diameter of the expandable part of the eSheath by a digital caliper. The most distal part $(0 \mathrm{~mm})$ is significantly smaller in diameter which is related to the absence of the plication of the dynamic expansion mechanism at the very distal part of the eSheath (Figures 2(a), 2(c), 3, and 5). After the insertion of the appropriate introducer, we started measuring at $30 \mathrm{~mm}$ at the distal end with $30 \mathrm{~mm}$ increments until the sleeve region (300 $\mathrm{mm}$ ) was reached (Figure 1(b)).
The second assessment aimed to determine maximum ex vivo changes induced by the pusher of the ECDS (Figure 2(b)). The pusher secures the position of the valve within the system during the passage through the sheath, and the diameter of the pusher is larger than the diameter of the crimped valve (Figure 2(b)). The respective ECDS for the $23 \mathrm{~mm}$ and $26 \mathrm{~mm}$ Sapien valves were used in the $14 \mathrm{~F}$ eSheath and the $29 \mathrm{~mm}$ valve ECDS in the $16 \mathrm{~F}$ eSheath. The balloon part of the ECDS was therefore locked outside the ECDS pusher part ( $n=5$ for each group).

Finally, we assessed the maximum outer dimensions of the eSheath following the in vivo TAVR implantation $(n=5$ for each group).

2.4. Statistical Analysis. Results are expressed as mean \pm standard deviation. The significance of variability among the means of the experimental groups was determined by 1- or 2-way ANOVA. All statistical tests were performed by using the software JMP (Version 7, SAS Institute Inc., Cary, NC, 1989-2007). Differences among experimental groups were considered statistically significant at $P<0.05$.

\section{Results}

The Edwards eSheath represents an ultralow delivery profile. The arterial sheath consists of a flexible part $(360 \mathrm{~mm})$ which is placed into the femoral artery $(\sim 270 \mathrm{~mm})$ except for the outer sleeve at the very proximal part $(\sim 90 \mathrm{~mm})$. A rigid 


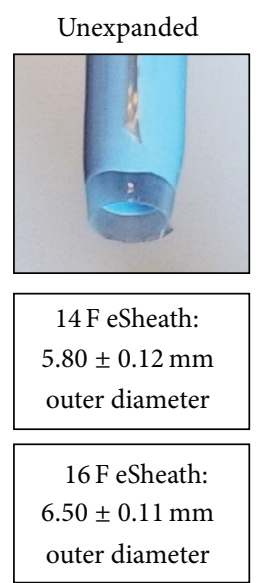

(a)

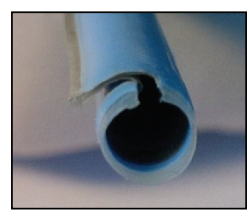

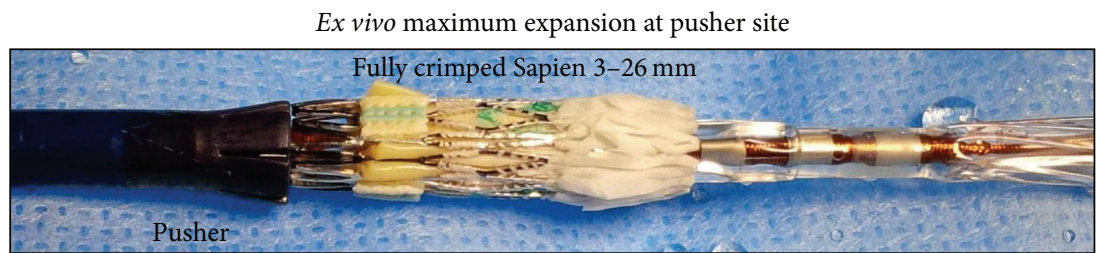

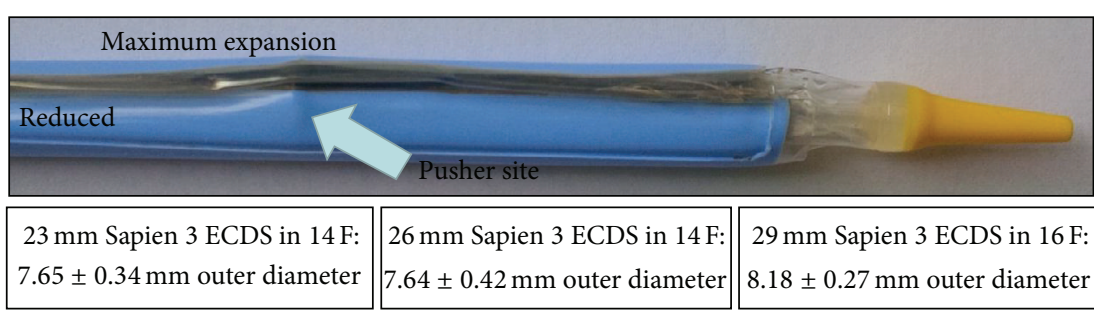

(b)

Reduced eSheath diameter post TAVI

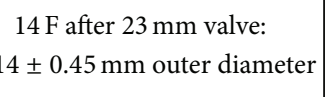

$14 \mathrm{~F}$ after $26 \mathrm{~mm}$ valve:

$7.26 \pm 0.40 \mathrm{~mm}$ outer diameter

$16 \mathrm{~F}$ after $29 \mathrm{~mm}$ valve:

$8.10 \pm 0.58 \mathrm{~mm}$ outer diameter

(c)

Figure 2: Outer diameter dimensions of the eSheath. (a) Outer diameter of the unexpanded $14 \mathrm{~F}$ eSheath was $5.80 \pm 0.12 \mathrm{~mm}$ and $6.50 \pm$ $0.11 \mathrm{~mm}$ for the $16 \mathrm{~F}$ eSheath. (b) Ex vivo maximum expansion diameter at the pusher site during the passage of the fully crimped $26 \mathrm{~mm}$ Sapien 3 valve. Maximum expansion diameter was $7.65 \pm 0.34 \mathrm{~mm}$ with a $23 \mathrm{~mm}$ Sapien ECDS in a $14 \mathrm{~F}$ eSheath, $7.64 \pm 0.42 \mathrm{~mm}$ in a $26 \mathrm{~mm}$ Sapien ECDS in a $14 \mathrm{~F}$ eSheath, and $8.18 \pm 0.27 \mathrm{~mm}$ in a $29 \mathrm{~mm}$ Sapien 3 ECDS in a $16 \mathrm{~F}$ eSheath. (c) Expanded distal end of the $14 \mathrm{~F}$ femoral eSheath after TAVR without the introducer. Outer eSheath diameter was $7.14 \pm 0.45 \mathrm{~mm}$ after implantation of a $23 \mathrm{~mm}$ valve in a $14 \mathrm{~F}$ eSheath, $7.26 \pm 0.40 \mathrm{~mm}$ after implantation of $26 \mathrm{~mm}$ valve in a $14 \mathrm{~F}$ eSheath, and $8.10 \pm 0.58 \mathrm{~mm}$ after implantation of a $29 \mathrm{~mm}$ valve in a $16 \mathrm{~F}$ eSheath.

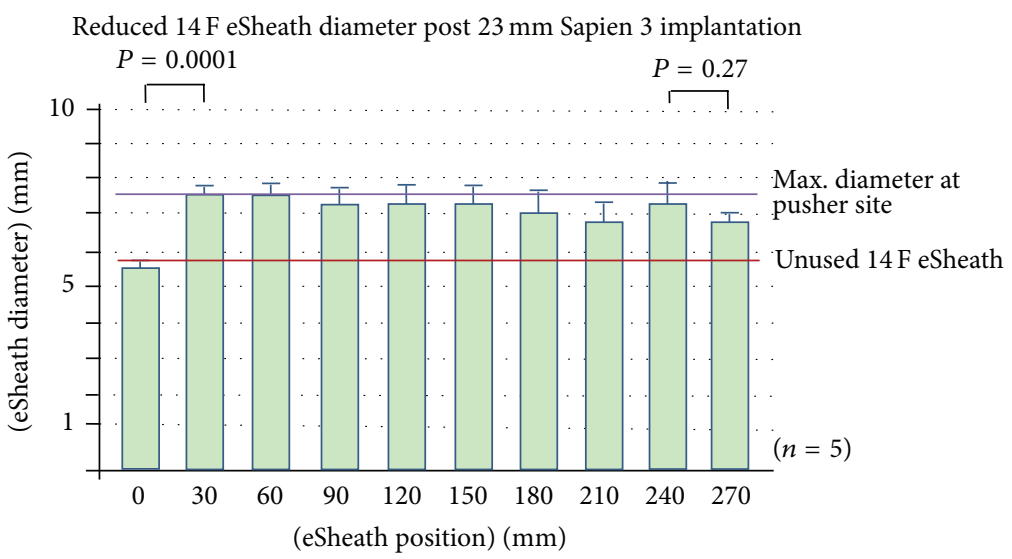

FIGURE 3: Longitudinal $14 \mathrm{~F}$ eSheath outer diameters at the 0, 30, 60, 90, 120 150, 180, 210, 240, and $270 \mathrm{~mm}$ measuring points following $23 \mathrm{~mm}$ Sapien 3 implantation.

proximal extracorporeal section includes the bleed back prevention mechanism of the eSheath (Figure 1(a)).

3.1. Assessment of Unused eSheaths. The outer diameters of the flexible part of the unused $14 \mathrm{~F}$ and $16 \mathrm{~F}$ eSheaths were measured at $5.80 \pm 0.12 \mathrm{~mm}$ and $6.50 \pm 0.11 \mathrm{~mm}$, respectively. Interestingly, these diameters of the unused control sheaths are already higher than the minimum arterial diameters of
$5.5 \mathrm{~mm}$ for the $14 \mathrm{~F}$ and $6.0 \mathrm{~mm}$ for the $16 \mathrm{~F}$ eSheath recommended by the manufacturer (Figure 1(b)).

3.2. Ex Vivo Assessment of the Maximum eSheath Expansion at the Pusher Site. Maximum enlargement of the eSheath was measured at the pusher site of the ECDS. Therefore, we locked the balloon outside the ECDS pusher and inserted the appropriate $23 \mathrm{~mm}$ and $26 \mathrm{~mm}$ ECDS in the $14 \mathrm{~F}$ eSheath and 


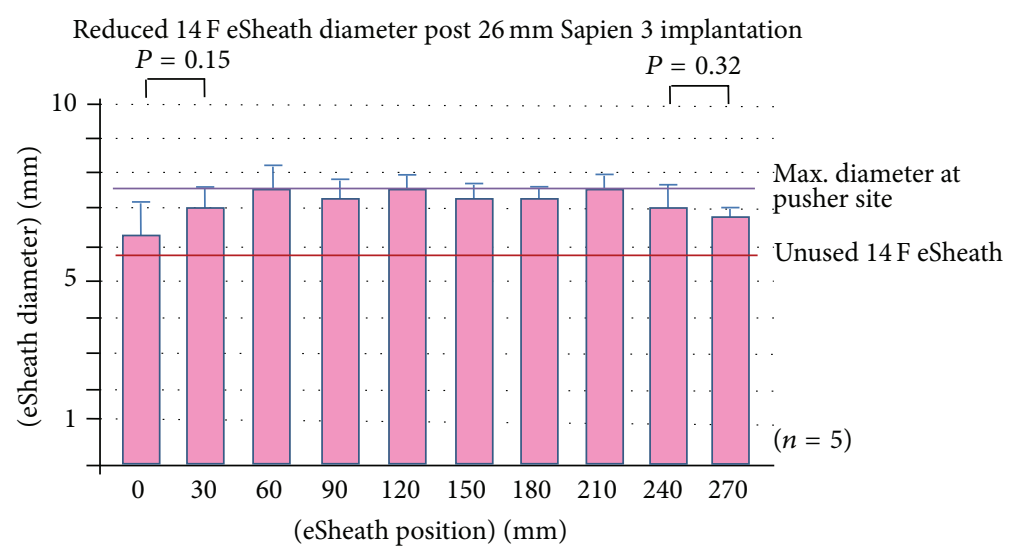

FIGURE 4: Longitudinal $14 \mathrm{~F}$ eSheath outer diameters at the $0,30,60,90,120150,180,210,240$, and $270 \mathrm{~mm}$ measuring points following $26 \mathrm{~mm}$ Sapien 3 implantation.

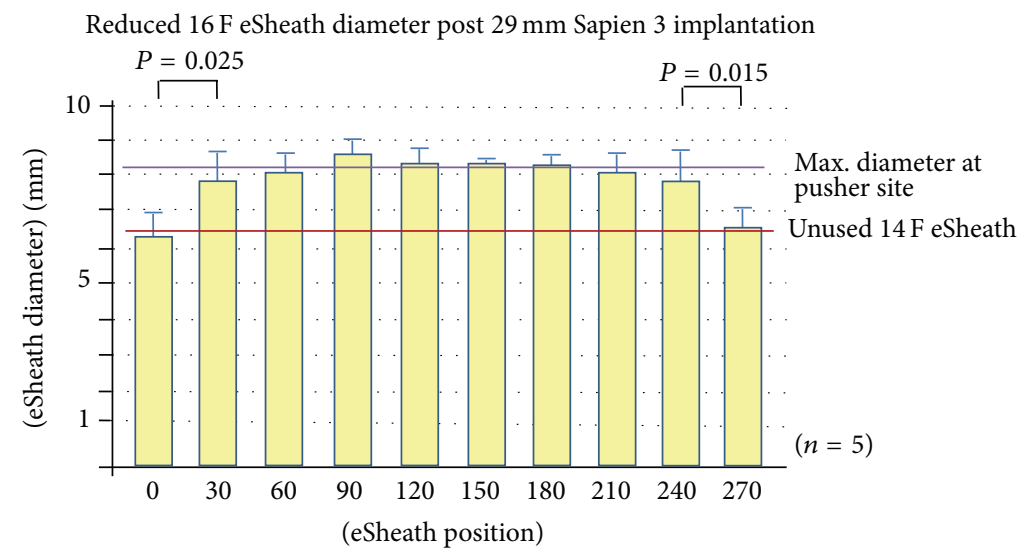

FIGURE 5: Longitudinal $16 \mathrm{~F}$ eSheath outer diameters at the 0, 30, 60, 90, 120 150, 180, 210, 240, and 270 mm measuring points following 29 mm Sapien 3 implantation.

the $29 \mathrm{~mm}$ ECDS into the $16 \mathrm{~F}$ eSheath. The pusher was placed into the appropriate eSheath at $60 \mathrm{~mm}$ from the distal expandable part. The maximum outer diameter was measured at the pusher site using a digital caliper (Figure 1).

The outer diameters of the eSheaths increased significantly after insertion of the ECDS pusher into the eSheath. Introduction of the $23 \mathrm{~mm}$ ECDS ( $7.0 \mathrm{~mm}$ pusher diameter) into the nominal $14 \mathrm{~F}$ eSheath led to a maximum eSheath dimension at the pusher site of $7.65 \mathrm{~mm}$, nominally corresponding to $\sim 23 \mathrm{~F}$. The $26 \mathrm{~mm}$ Edwards Commander Delivery System (7.0 $\mathrm{mm}$ pusher diameter) showed a comparable maximum eSheath dimension at the pusher site of $7.64 \mathrm{~mm}$, approximately $23 \mathrm{~F}$. Finally, the advancement of the $29 \mathrm{~mm}$ Edwards Commander Delivery System $(8.0 \mathrm{~mm}$ pusher diameter) into the $16 \mathrm{~F}$ eSheath led to a maximum eSheath dimension at the pusher site of $8.18 \mathrm{~mm}$, approximately $24.5 \mathrm{~F}$.

3.3. Assessment of the eSheath Dimensions after Sapien 3 Femoral Transcatheter Valve Implantation. We collected five $14 \mathrm{~F}$ eSheaths after $23 \mathrm{~mm}$ Sapien 3 implantation, five $14 \mathrm{~F}$ eSheaths after $26 \mathrm{~mm}$ Sapien 3 implantation, and five $16 \mathrm{~F}$
eSheaths after $29 \mathrm{~mm}$ Sapien 3 implantation. Multislice computed tomography (MSCT) of the iliac and femoral artery revealed mean minimal luminal diameter (MLD) of $7.64 \pm$ $0.75 \mathrm{~mm}$ in the Sapien $23 \mathrm{~mm}$ group, $7.70 \pm 0.87 \mathrm{~mm}$ in the Sapien $26 \mathrm{~mm}$ group (both $14 \mathrm{~F}$ eSheaths), and $8.48 \pm 0.60 \mathrm{~mm}$ in the Sapien $29 \mathrm{~mm}$ group using $16 \mathrm{~F}$ eSheaths $(P=\mathrm{NS}$ for all comparisons). TAVI implantation was successful in all cases, without relevant femoral access site adverse events. The outer diameters of the eSheaths increased significantly after Sapien 3 implantation compared to the diameters mentioned above for the control sheaths. The mean outer diameter of the $14 \mathrm{~F}$ eSheath after $23 \mathrm{~mm}$ valve implantation was $7.14 \pm 0.45 \mathrm{~mm}$ and that of the $14 \mathrm{~F}$ eSheath after placing the $26 \mathrm{~mm}$ valve was $7.26 \pm 0.40 \mathrm{~mm}$ and it was markedly larger with $8.10 \pm$ $0.58 \mathrm{~mm}$ for the $16 \mathrm{~F}$ eSheath after $29 \mathrm{~mm}$ valve implantation ( $P<0.02$ for all used eSheaths compared to unused eSheaths) (Figures 3-5).

Interestingly, the $14 \mathrm{~F}$ eSheath showed a trend to larger outer diameters after using the $26 \mathrm{~mm}$ valve compared to the $23 \mathrm{~mm}$ valve. However, this was not statistically significant $(P=0.6)$. The $16 \mathrm{~F}$ eSheath showed a significant larger outer 
TABLE 1: Summary of unexpanded, maximum, and mean eSheath outer diameters of $14 \mathrm{~F}$ and $16 \mathrm{~F}$ eSheaths.

\begin{tabular}{lcccc}
\hline eSheath outer diameter $(\mathrm{mm})$ & $\begin{array}{c}\text { 14 F eSheath } / 23 \mathrm{~mm} \\
\text { Sapien }\end{array}$ & $\begin{array}{c}14 \mathrm{~F} \text { eSheath/26 mm } \\
\text { Sapien }\end{array}$ & $\begin{array}{c}\text { 16 F eSheath/29 mm } \\
\text { Sapien }\end{array}$ & $\begin{array}{c}P \text { value } \\
(23 \mathrm{versus} \mathrm{29} \mathrm{mm})\end{array}$ \\
\hline Unexpanded eSheath & $5.80 \pm 0.12 \mathrm{~mm}$ & $5.80 \pm 0.12 \mathrm{~mm}$ & $6.50 \pm 0.11 \mathrm{~mm}$ & 0.0001 \\
eSheath ex vivo max. at pusher site & $7.65 \pm 0.34 \mathrm{~mm}$ & $7.64 \pm 0.42 \mathrm{~mm}$ & $8.18 \pm 0.27 \mathrm{~mm}$ & 0.03 \\
Mean eSheath diameter after TAVI & $7.14 \pm 0.45 \mathrm{~mm}$ & $7.26 \pm 0.40 \mathrm{~mm}$ & $8.10 \pm 0.58 \mathrm{~mm}$ & 0.02 \\
\hline
\end{tabular}

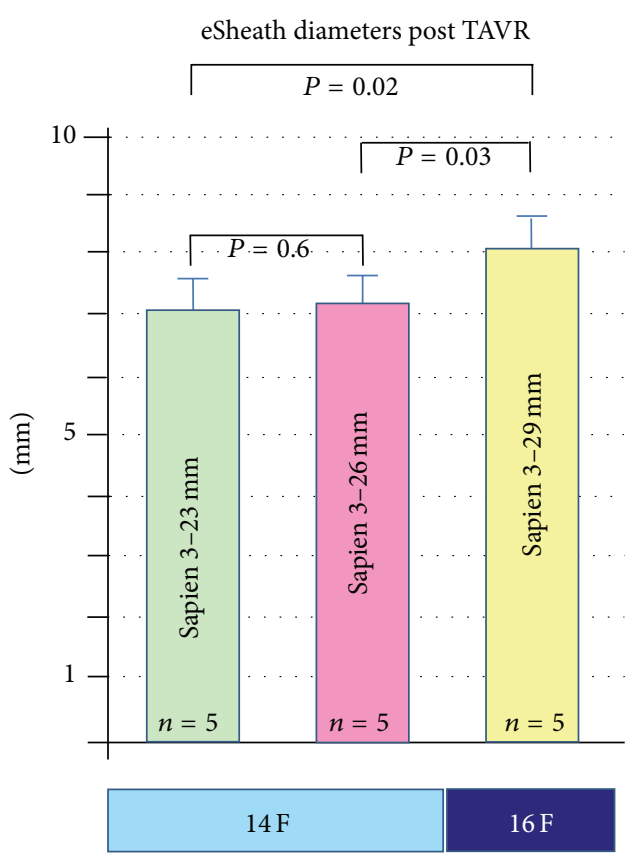

FIgURE 6: Mean outer diameters of the $14 \mathrm{~F}$ eSheath after Sapien $23 \mathrm{~mm}$ and $26 \mathrm{~mm}$ implantation, respectively, and of the $16 \mathrm{~F}$ eSheath after Sapien $29 \mathrm{~mm}$ implantation.

diameter after $29 \mathrm{~mm}$ valve implantation compared to the $14 \mathrm{~F}$ eSheath $(P=0.03)$ (Figure 6).

The circumferential plastic sleeve stabilized the expandable part of the eSheath at the intersection between the expandable part and the stiff bleed back prevention valve (Figures 1(a) and 5). The outer diameter of the expandable part without the sleeve $(0-240 \mathrm{~mm})$ of the $16 \mathrm{~F}$ eSheath after $29 \mathrm{~mm}$ valve implantation was significantly smaller than the adjacent sleeve area $(270 \mathrm{~mm})$ (Figures $3-5)$. This trend was also noted in the $14 \mathrm{~F}$ eSheath after $23 \mathrm{~mm}$ and $26 \mathrm{~mm}$ valve implantation, however, without reaching statistical significance.

The area of the expandable part of the $16 \mathrm{~F}$ eSheath after $29 \mathrm{~mm}$ valve implantation was significantly smaller than the adjacent $24 \mathrm{~cm}$ measurement at the expandable part without the sleeve (Figures 3-5). This trend was also noted in the $14 \mathrm{~F}$ eSheath after $23 \mathrm{~mm}$ and $26 \mathrm{~mm}$ valve implantation, however, without reaching statistical significance. In summary, the eSheath outer diameters are about $1.2 \mathrm{~mm}$ larger compared to the nominal inner diameter and increase by another $1.7 \mathrm{~mm}$ during valve passage (Table 1). Following real-world valve implantation, the observed eSheath retraction after removal is $0.1-0.5 \mathrm{~mm}$.

\section{Discussion}

Transcatheter aortic valve replacement (TAVR) represents a valid option for the treatment of severe symptomatic aortic stenosis in patients at very high or prohibitive surgical risk [7]. However, vascular complications still represent a challenging limitation in transfemoral transcatheter aortic valve replacement due to significant morbidity and mortality [4-7]. These may include major and minor VARC complications, for example, aortic dissection, access site bleeding, femoral dissection, and vessel occlusion [8-11].

The introduction of lower-profile eSheaths with a dynamic expansion mechanism leads to a significant reduction of vascular complications after transfemoral TAVR [2]. This improvement enabled TAVR in a wider population including patients with diseased peripheral arteries.

The eSheath is distributed with a nominal inner lumen diameter of $14 \mathrm{~F}$ for the application of the $23 \mathrm{~mm}$ and $26 \mathrm{~mm}$ Sapien 3 valves and $16 \mathrm{~F}$ for the application of the $29 \mathrm{~mm}$ Sapien 3 valve. With a dynamic expansion mechanism, this sheath system adapts transiently during the passage of the Edwards Sapien 3 transcatheter heart valve and abates to a lower-profile diameter thereafter.

eSheath dimensions are suggested to be about $16-18 \mathrm{~F}$ for TAVR implantation, but the outer diameters are about $1.2 \mathrm{~mm}$ larger. These increase by another $1.7 \mathrm{~mm}$ during valve passage. However, significantly larger dimensions are reached at the site of the pusher during implantation, although this is limited to a short period of time during valve passage.

In addition, the eSheath does not retract fully to the native dimensions after valve passage, as seen after complete assessment of the used eSheaths. The retraction of mean eSheath outer diameter after removal observed in our study was on average $0.5 \mathrm{~mm}$ for the $14 \mathrm{~F}$ eSheath and only $0.1 \mathrm{~mm}$ for the $16 \mathrm{~F}$ eSheath. This suggests that the expansion following passage of the large $29 \mathrm{~mm}$ valve is less reversible compared to the smaller valves (Figure 6).

The introduction of lower-profile sheaths has undoubtedly improved vascular complications rates as a major limitation of the TAVR procedure. However, we believe that the TAVR community may have a particular interest in knowing the effective outer eSheath diameters during valve advancement to further improve patient outcome.

Limitations. We did not measure the loaded Sapien 3 valve within the eSheath ex vivo to avoid any contamination or damage of the valve/system prior to implantation. 


\section{Conclusion}

Nominal $14 \mathrm{~F}$ and $16 \mathrm{~F}$ luminal eSheath systems showed significant outer diameter changes during and after advancement of the appropriate Edwards Commander Delivery System. This information may be of significant value for patient selection suggested for TAVR.

\section{Abbreviations}

DEM: Dynamic expansion mechanism

ECDS: Edwards Commander Delivery System

F: $\quad$ French

LPS: Low-profile sheath

TAVR: Transcatheter aortic valve replacement

TAVI: Transcatheter aortic valve implantation

MSCT: Multislice computed tomography

MLD: Minimal luminal diameter.

\section{Conflict of Interests}

The authors declare that there is no conflict of interests regarding the publication of this paper.

\section{Authors' Contribution}

Till Koehler and Michael Buege contributed equally to this work. Klaus Tiroch and Marc Vorpahl shared senior author contribution.

\section{References}

[1] M. Freeman, J. Rodés-Cabau, M. Urena et al., "First-in-man transfemoral transcatheter aortic valve replacement with the $29 \mathrm{~mm}$ Edwards SAPIEN XT Valve," Catheterization and Cardiovascular Interventions, vol. 82, no. 4, pp. 664-670, 2013.

[2] M. Barbanti, R. K. Binder, M. Freeman et al., "Impact of lowprofile sheaths on vascular complications during transfemoral transcatheter aortic valve replacement," EuroIntervention, vol. 9, no. 8, pp. 929-935, 2013.

[3] B. Borz, E. Durand, C. Tron et al., "Expandable sheath for transfemoral transcatheter aortic valve replacement: procedural outcomes and complications," Catheterization and Cardiovascular Interventions, vol. 83, no. 6, pp. E227-E232, 2014.

[4] R. Lange, S. Bleiziffer, D. Mazzitelli et al., "Improvements in transcatheter aortic valve implantation outcomes in lower surgical risk patients: a glimpse into the future," Journal of the American College of Cardiology, vol. 59, no. 3, pp. 280-287, 2012.

[5] A. P. Kappetein, S. J. Head, P. Généreux et al., "Updated standardized endpoint definitions for transcatheter aortic valve implantation: the valve academic research consortium-2 consensus document," Journal of the American College of Cardiology, vol. 60, no. 15, pp. 1438-1454, 2012.

[6] H. B. Ribeiro, J. G. Webb, R. R. Makkar et al., "Predictive factors, management, and clinical outcomes of coronary obstruction following transcatheter aortic valve implantation: insights from a large multicenter registry," Journal of the American College of Cardiology, vol. 62, no. 17, pp. 1552-1562, 2013.

[7] M. B. Leon, C. R. Smith, M. Mack et al., "Transcatheter aorticvalve implantation for aortic stenosis in patients who cannot undergo surgery," The New England Journal of Medicine, vol. 363, no. 17, pp. 1597-1607, 2010.

[8] K. Hayashida, T. Lefvre, B. Chevalier et al., "Transfemoral aortic valve implantation: new criteria to predict vascular complications," JACC: Cardiovascular Interventions, vol. 4, no. 8, pp. 851-858, 2011.

[9] A. Nagasawa, S. Shirai, M. Hanyu, Y. Arai, N. Kamioka, and M. Hayashi, "Descending aortic dissection injured by tip of the sheath during transcatheter aortic valve implantation," Cardiovascular Intervention and Therapeutics, 2015.

[10] S. Toggweiler, J. Leipsic, R. K. Binder et al., "Management of vascular access in transcatheter aortic valve replacement: part 2: vascular complications," JACC: Cardiovascular Interventions, vol. 6, no. 8, pp. 767-776, 2013.

[11] N. M. van Mieghem, D. Tchetche, A. Chieffo et al., "Incidence, predictors, and implications of access site complications with transfemoral transcatheter aortic valve implantation," The American Journal of Cardiology, vol. 110, no. 9, pp. 1361-1367, 2012. 


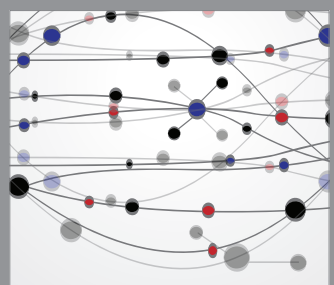

The Scientific World Journal
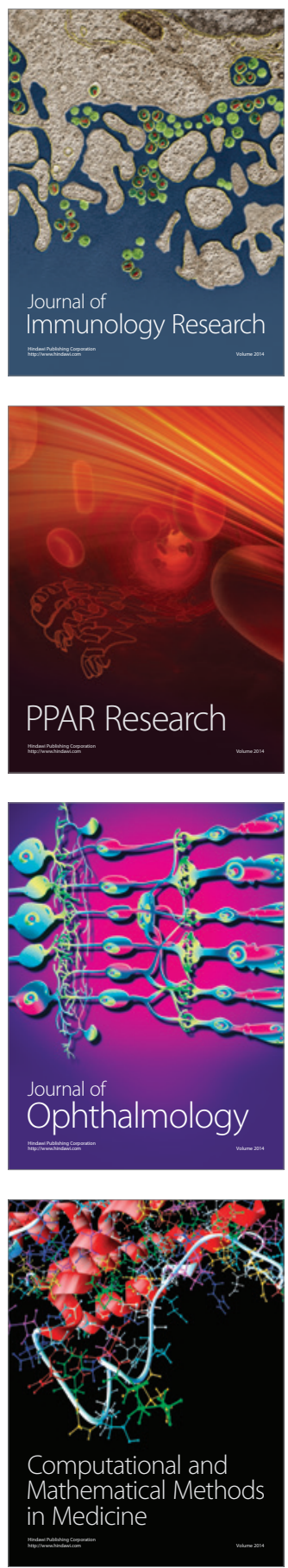

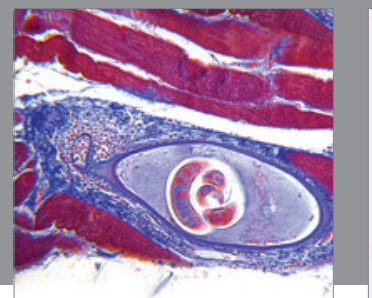

Gastroenterology

Research and Practice
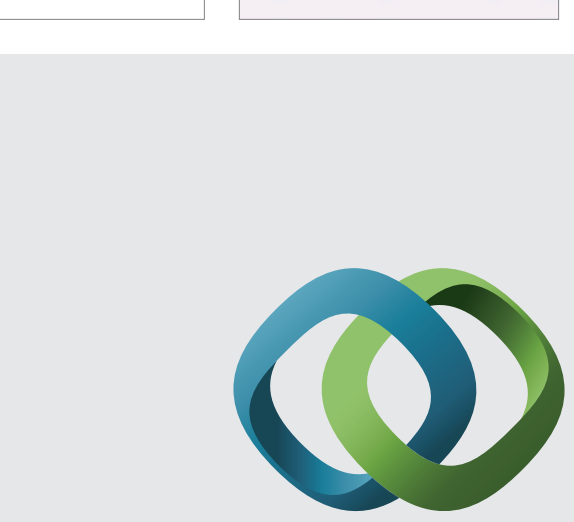

\section{Hindawi}

Submit your manuscripts at

http://www.hindawi.com
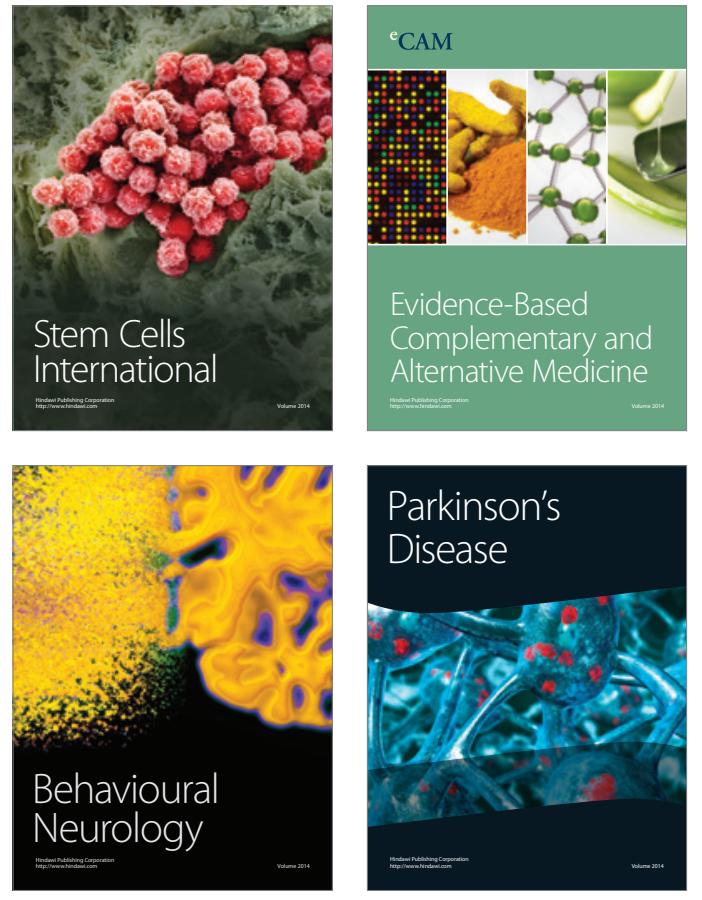
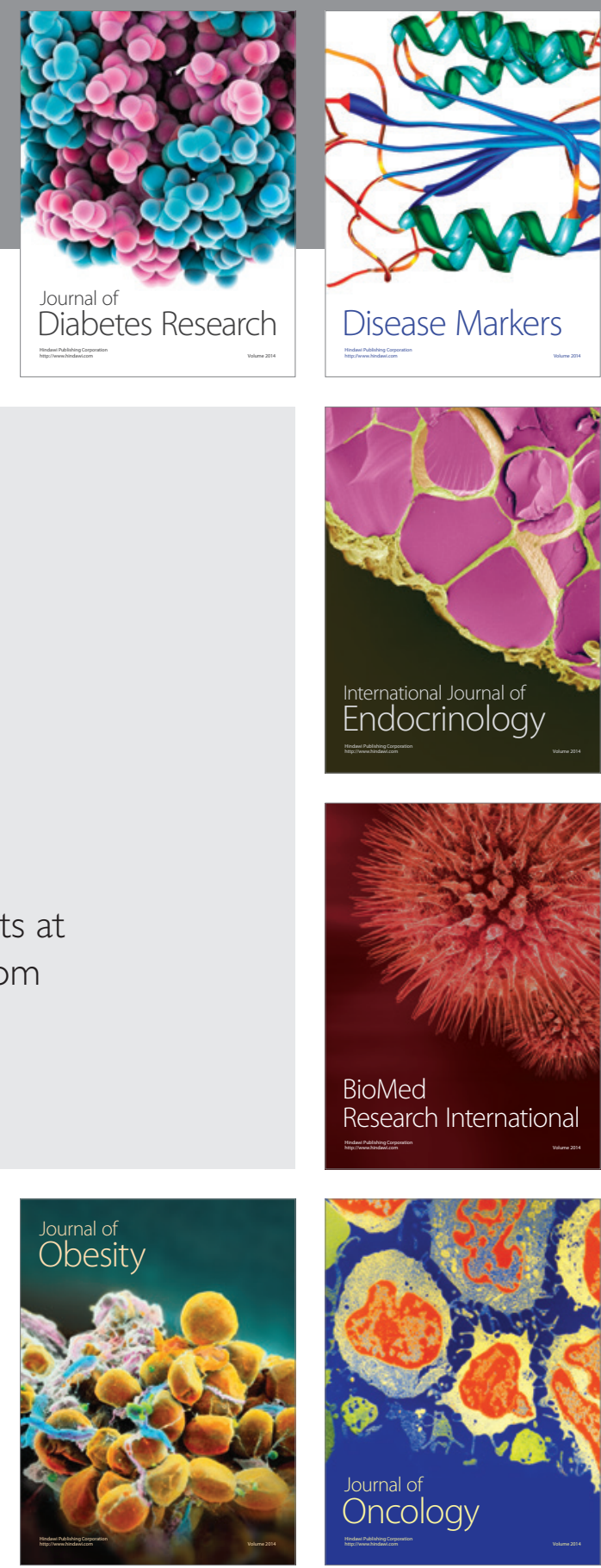

Disease Markers
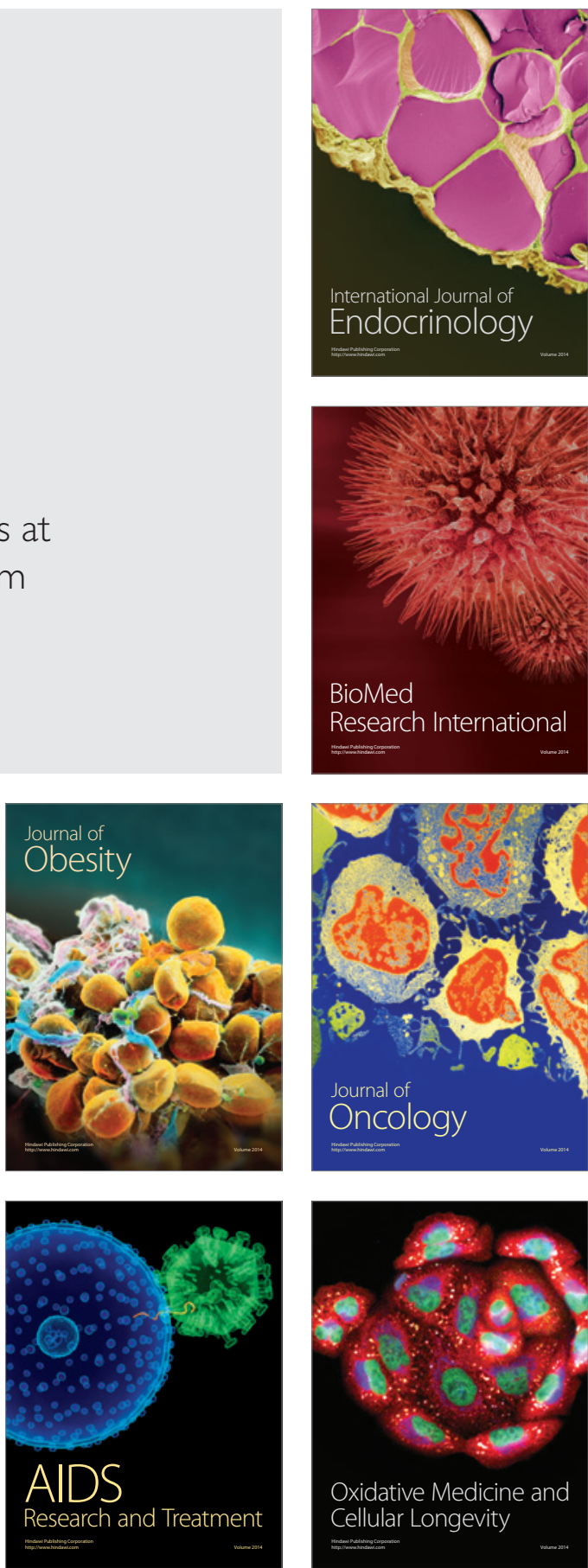